\title{
GASTO PÚBLICO TOTAL Y SOCIAL: EL CASO DE URUGUAY EN EL SIGLO XX*
}

\author{
PAOLA AZAR \\ Universidad de la República (Uruguay) ${ }^{\text {a }}$ \\ SEBASTIÁN FLEITAS \\ Universidad de la República (Uruguay) ${ }^{\text {a }}$
}

Total and social public expenditure: the Uruguayan case in the 20th century

\begin{abstract}
This article discusses the evolution of the total and social public expenditure in Uruguay during the $20^{\text {th }}$ century. It analyzes the growth path of the social public expenditure and the extent up to which it could be preserved from the cyclical economic downturns and the fiscal constraints of the Public Sector. The paper finds a low long-run elasticity of public spending to GDP leading to a slow growth of social public expenditure and a remarkable procyclical pattern of total and social public expenditure. It also shows that social spending, especially education expenditure, has often been used as an instrument to curb budget deficits. No distinctive «fiscal regimes» for the period could be identified.
\end{abstract}

Keywords: social expenditure, fiscal policy, macroeconomics, economic history

JEL Classification: N16, E61, I38

* Received 8 April 2011. Accepted 20 September 2011. Los autores agradecen los comentarios recibidos en la sesión "Latin American economic backwardness revisited. New empirical contributions» del XV Congreso Mundial de Historia Económica realizado en Utrecht (Holanda), en la mesa de Distribución del Ingreso de las XXIV Jornadas Anuales de Economía del Banco Central del Uruguay y en las VI Jornadas de Historia Económica de la Asociación Uruguaya de Historia Económica. También agradecen a los árbitros anónimos las sugerencias y observaciones que permitieron mejorar significativamente el trabajo original. Los errores y omisiones que persistan son de entera responsabilidad de los autores. Por último, los autores reconocen el apoyo recibido de la Comisión Sectorial de Investigación Científica de la Universidad de la República, a través de su Programa de Iniciación a la Investigación.

a Área de Historia Económica, Instituto de Economía, Facultad de Ciencias Económicas y de Administración. J. Requena, 1375, Montevideo. pazar@iecon.ccee.edu.uy; sefle@iecon.ccee.edu.uy. 


\section{RESUMEN}

Este trabajo describe la evolución del gasto público total y social de Uruguay en el siglo XX. Indaga sobre el ritmo en que el Estado destinó recursos al incremento del gasto social y sobre la medida en que logró preservarlo frente a las fluctuaciones cíclicas de la economía y los problemas de financiación del Sector Público. Los resultados muestran que la elasticidad del gasto público respecto al producto fue especialmente baja en Uruguay, lo que redujo la expansión del gasto social y que el perfil procíclico del gasto total y social se acentuó en el período. También se muestra que el gasto social, en particular la partida destinada a educación, con frecuencia se utilizó como instrumento de ajuste fiscal. No lograron identificarse comportamientos asociados a diferentes «regímenes fiscales» durante el período.

Palabras clave: gasto social, política fiscal, macroeconomía, historia económica

\section{INTRODUCCIÓN}

Uruguay se destaca en el ámbito regional debido a la temprana emergencia de un sistema de protección social de corte estatal que se consolidó en el siglo XX y que, aún en la actualidad, lo ubica entre los más avanzados, considerando a los países de renta media.

Este trabajo analiza la evolución del gasto público total, en educación, salud y seguridad social (en adelante, "gasto social») entre 1905 y 2000 para el caso uruguayo. Busca conocer cuánto de las prioridades establecidas en materia social pudo mantenerse ante las presiones derivadas de las fluctuaciones cíclicas de la economía y las necesidades de equilibrar las cuentas públicas. Además, considerando si las decisiones sobre estos aspectos variaron por subperíodos, se contrasta la hipótesis de existencia de diferentes «regímenes fiscales» durante esos años.

El abordaje combina diferentes herramientas metodológicas. La relación de largo plazo entre el gasto y el producto y sus implicancias para el crecimiento del gasto social se aproxima a través de un análisis de cointegración. El resultado se compara con el de países que presentan similitudes con Uruguay. El patrón cíclico y la volatilidad de las partidas fiscales se examinan a partir de la correlación cruzada entre los componentes cíclicos de las series y sus desvíos estándar. Finalmente, se identifican etapas de restricción y flexibilidad en las necesidades de financiamiento del Gobierno Central y se observa el comportamiento de las partidas sociales en cada una de ellas.

Pese a la permanencia de la matriz de protección social uruguaya, los resultados muestran que el crecimiento del gasto público total fue muy 
modesto en el siglo y que la gestión del gasto social, en el largo plazo, no logró preservarse explícitamente de los desequilibrios e inestabilidades a los que estuvo sometida la política fiscal.

El análisis se estructura de la siguiente manera: en el primer apartado se presentan el marco conceptual y los antecedentes del estudio; en el segundo se detallan las fuentes de datos y las características de las series de gasto. En el apartado 3 se discute la relación de largo plazo entre gasto público y producto en Uruguay, contrastando con la evidencia internacional. En los apartados siguientes se revisa el patrón cíclico del gasto público y de sus partidas sociales y su reacción ante fases de «desajuste» y «ajuste» fiscal. En el apartado 6 se discute la hipótesis de existencia de diferentes «regímenes fiscales» en el siglo, a la luz de la evidencia. El último apartado resume los hallazgos del trabajo.

\section{LA EVOLUCIÓN DEL GASTO PÚBLICO: REVISIÓN DE ANTECEDENTES}

Este análisis de la trayectoria del gasto público y social en Uruguay enfoca tres aspectos específicos: su vínculo con el crecimiento económico de largo plazo, su asociación con los ciclos del producto y su reacción ante las restricciones de financiamiento del Estado.

La existencia de una relación de largo plazo entre el gasto público y el producto ha sido ampliamente debatida en la literatura económica. Inicialmente propuesta por Wagner (1912), la idea de una asociación positiva entre el crecimiento de las economías y la expansión del sector público motivó una larga serie de trabajos interesados en identificar y discutir esta regularidad, que terminaría conociéndose como «ley de Wagner». Aunque originalmente el autor no aludió a una relación causa-efecto entre ambas variables, un importante conjunto de estudios ha procurado determinar la dirección de la influencia (Peacock y Scott 2000 y luego Chang 2002 realizan una revisión y discusión de la literatura sobre el tema).

La relación entre gasto y producto en América Latina ha sido explorada en varios estudios (Murthy 1993, Hayo 1994 y Díaz Fuentes 1994 para México, Brasil y Argentina; Thornton 1998 para Argentina, Brasil y Chile; Akitoby et al. 2006 y Clements et al. 2007 para varios países). Sin embargo, en todos los casos se consideran períodos comprendidos entre los años setenta y finales del siglo XX o tramos cortos de etapas anteriores. Sólo en Comín et al. (2008) se estudian los casos de Argentina, Brasil, México y España, para el período 1900-2000. Encuentran una elasticidad entre gasto y producto mayor a la unidad y verifican la causalidad en el sentido planteado por la «ley de Wagner».

A este debate sobre la relación de largo plazo se suman preguntas sobre los vínculos de corto plazo entre gasto público y producto. Pese a los consensos 
teóricos del lado neoclásico y keynesiano sobre la conveniencia de un manejo fiscal neutral al ciclo o incluso contracíclico, las prácticas no parecen ir en ese sentido. La información disponible desde la década de 1970 ha permitido constatar que las economías latinoamericanas han presentado altos niveles de prociclicidad en relación con las desarrolladas y con otras emergentes (Gavin et al. 1996, Gavin y Perotti 1997, Martner y Tromben 2003, Akitoby et al. 2004, Kaminsky et al. 2004, Martner y Aldunate 2006, Ilzetzki y Vegh 2008). No obstante, debido a la escasez de información, no ha sido posible establecer si este patrón de la política fiscal regional se presentaba en períodos anteriores.

La prociclicidad ha sido explicada enfatizando las cambiantes condiciones de acceso a los mercados internacionales de crédito (Aizenman et al., 1996, y Gavin y Perotti 1997a y 1997b) o las presiones políticas de diferentes orígenes durante el ciclo económico (Talvi y Vegh 2000, Lane 2003, Lane y Tornell 1999 y Alesina et al. 2007). En el caso de América Latina, Ffrench-Davis (2003) subraya que la prociclicidad se explica porque los países fijan metas fiscales sin tener en cuenta el momento del ciclo económico, lo que provoca que el gasto del sector público se financie con ingresos transitorios. CEPAL (2007) atribuye el manejo procíclico a las decisiones de los gobiernos que necesitan adaptarse a las demandas sociales que resurgen en tiempos de recuperación, luego de haber sido postergadas durante las crisis o recesiones.

Otro de los rasgos típicos de las finanzas públicas latinoamericanas tiene que ver con las fuertes restricciones de financiamiento que enfrentan. Ello hace que las economías sean altamente dependientes del mercado de capitales. Esta característica las expone a ajustes fiscales recurrentes, que afectan tanto al gasto público -en todos sus componentes- como a la estructura tributaria, más allá de la estrategia contracíclica que pueda haberse diseñado (Ocampo 2002).

En términos del gasto público social, diversos estudios para la región han encontrado prociclicidad, sobre todo en el gasto en educación y salud; en tanto, la seguridad social parecería tener un desempeño acíclico. Por su parte, ante crisis fiscales, este tipo de gasto ha tendido a ajustarse a la baja (Cominetti 1994). Los trabajos se han concentrado en la dinámica desde las décadas de 1980 y 1990 (Hicks y Wodon 2001, Martner y Aldunate 2006, Martínez y Espíndola 2007 y CEPAL 2007); o en la información disponible entre 1970 y 1997 (Wibbels 2006 y Segura-Ubiergo 2007).

Para el caso de Uruguay, la literatura brinda evidencia de diferente alcance sobre los aspectos que se propone abordar este trabajo. La economía uruguaya se ha caracterizado por su elevada volatilidad cíclica en el largo plazo (Kamil y Lorenzo 1998, Bértola y Lorenzo 2004, Cal 2004, y Carbajal y De Melo 2007). Según Oddone (2005), las instituciones, la calidad de las políticas (entre ellas la fiscal) y el comportamiento de los gobernantes contribuyeron a profundizar los ciclos durante el siglo XX. También se ha estudiado el perfil procíclico de la política fiscal durante la segunda mitad del siglo (Stein et al. 1998, Mailhos y Sosa 1999 y 2000). Para el siglo XX, a partir de la evolución del gasto, los tributos, los resultados fiscales y el endeudamiento 
público, una serie de investigaciones en historia económica identifica tres "regímenes fiscales», caracterizados por desempeños, resultados y expectativas distintas en el diseño de política (Azar et al. 2009).

En el marco de estos antecedentes regionales y locales, este trabajo aspira a brindar nueva evidencia que contribuya a la caracterización de la gestión del gasto público total y social para Uruguay, con una perspectiva de largo plazo.

\section{GASTO TOTAL Y SOCIAL EN URUGUAY: FUENTES Y TENDENCIAS}

Los datos sobre gasto total hacen referencia a la ejecución presupuestal del Gobierno Central y a sus resultados fiscales entre 1905 y 2000. Para describir el gasto social se contó con información desde 1910 hasta 2000. Se tomaron las partidas destinadas a educación y salud, que son parte del Gobierno Central, y a seguridad social (prestaciones a activos y pasivos), que son cubiertas por organismos autónomos. La consideración conjunta de lo gastado en seguridad social y lo ejecutado por el Gobierno Central (GC) da lugar al gasto del Gobierno Central Consolidado (GCC) ${ }^{1}$.

Las series utilizadas fueron tomadas de Azar et al. (2009). En el caso de educación y salud, se recurrió además a las siguientes fuentes: Acevedo (varios años), MSP-OPS (1972), BROU (1965), CGN (1996) y Corbo et al. (1989) ${ }^{2}$.

El Producto Bruto Interno (PBI) para todo el siglo fue tomado de Bertino y Tajam (1999), BROU (1965) y BCU (varios años). El PBI y las variables fiscales se consideraron a precios constantes. En el caso de estas últimas, se deflactaron por una serie de precios estimada a partir de Bértola et al. (1999), actualizada con datos del Instituto Nacional de Estadísticas. La serie de población fue tomada de Nahum (2006) y del Programa de Población, Unidad Multidisciplinaria, Facultad de Ciencias Sociales, UDELAR.

Durante el período, el gasto del GCC y el producto, en términos reales, siguieron una trayectoria muy cercana y sus variaciones estuvieron estrechamente asociadas (gráfico 1). En promedio, la tasa de crecimiento acumulativa anual del PBI fue del 2,6\% y la del gasto del GCC del 1,3\%. Se observa, no obstante, una volatilidad más elevada de este último, que tendió a acentuarse en la segunda mitad del siglo. En el cuadro A-1 se presentan indicadores de resumen de la dinámica de las series.

El gasto del GCC en relación a la población se multiplicó por 4 y respecto al producto, pasó del 12 al 38,1\% entre 1903 y 2000. Este ratio tomando el gasto del GC partió del 11\% y alcanzó el 23\% en 2000. Por su parte, la

\footnotetext{
1 Para estimar el gasto del Gobierno Central Consolidado se tomó el gasto del Gobierno Central neto de transferencias a los organismos previsionales. Para no incurrir en duplicaciones, este total es el que se suma a lo gastado en seguridad social (que ya tiene incluido el monto de transferencias).

2 Desde 1968, el gasto en salud consideró lo correspondiente a seguros de salud financiados por trabajadores formales y sus empleadores.
} 


\section{GRÁFICO 1}

EVOLUCIÓN DEL GASTO PÚBLICO DEL GOBIERNO CENTRAL CONSOLIDADO Y DEL PRODUCTO

$($ Precios constantes. Índice $100=1903)$

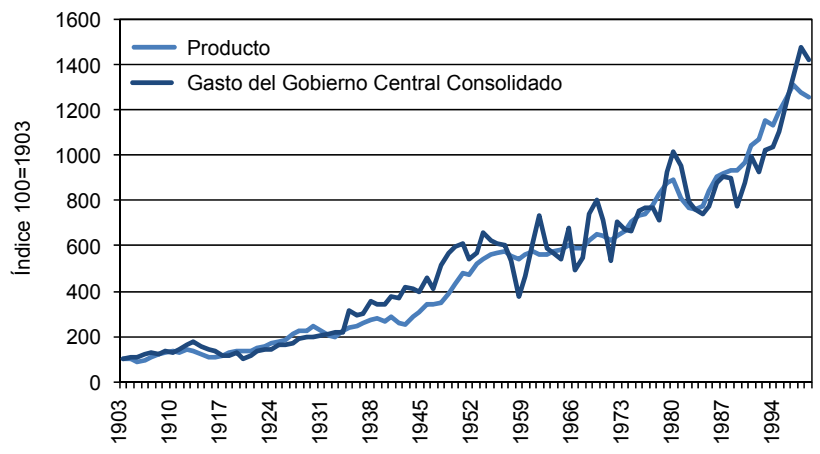

ponderación de las partidas de educación, salud y seguridad social en el producto se multiplicó por 8,7: creció del 2,5 al 21,8\% entre el principio y el final del período (gráfico 2).

Como muestra el gráfico 2, hasta la década de 1950, salud y educación, por un lado, y seguridad social, por otro, tenían participaciones similares. Desde entonces, la ponderación de seguridad social fue cubriendo porciones crecientes de ese total, hasta constituir alrededor del $70 \%$.

\section{CRECIMIENTO DEL GASTO PÚBLICO Y SOCIAL EN EL LARGO PLAZO}

La condición pionera de Uruguay en materia del desarrollo de su matriz de protección social pública hace interesante conocer cuál fue la prioridad fiscal que recibieron las áreas sociales en el tiempo. Dicha prioridad está influida, entre otras cuestiones, por el nivel de crecimiento económico del país y por el ritmo de expansión del gasto total por cada punto de crecimiento de la riqueza. Para conocer estas influencias, se estimó la elasticidad o relación entre el gasto público del GC y el producto entre 1903 y 2000, de acuerdo a los trabajos inspirados en la discusión de la «ley de Wagner». A efectos de contrastar los resultados obtenidos, el cálculo también se realizó para otros países.

Debido a la escasa disponibilidad de datos para el período bajo estudio, no pudo contarse con una muestra amplia de países. Por tanto, se seleccionó un conjunto de economías que permitieran controlar diferentes características del caso uruguayo. Por un lado, Argentina, Chile y Brasil, ya que comparten 


\section{GRÁFICO 2}

PARTICIPACIÓN DE LAS PARTIDAS DE GASTO SOCIAL EN EL PRODUCTO

(En porcentaje del PBI)



Fuentes: Acevedo (varios años); Azar et al. (2009), Bertino y Tajam (1999), Bértola et al. (1999), BROU (1965), BCU (varios años), CGN (1996); Corbo et al. (1989); INE; MSP-OPS (1972); BROU (1965).

con Uruguay el desarrollo temprano de sus sistemas de protección social y el alto esfuerzo del sector público en materia de bienestar, respecto a la región (Mesa-Lago, 1986, Filgueira 1998 y Segura-Ubiergo 2007). Luego, España e Italia por tratarse de economías con niveles de ingreso per cápita similares (incluso menores) a los de las economías latinoamericanas más avanzadas a principios de siglo (de hecho, Italia comenzó a converger con los países europeos más ricos tras la segunda posguerra y España recién en la década del sesenta). Finalmente, Canadá y Nueva Zelanda, que junto a Argentina, Chile y Uruguay, además de Estados Unidos y Australia son "economías de nuevo asentamiento”. Aunque en el transcurso del siglo XX cada una protagonizó trayectorias propias de éxitos y fracasos, al comienzo del período tenían condiciones similares en cuanto a la recepción de inmigración y la configuración de su estructura productiva (con importante participación de recursos naturales), así como niveles de riqueza próximos a los de los países europeos más avanzados (Álvarez et al. 2007).

La participación del gasto del GC en el producto para la primera década del siglo XX alcanzaba el 16,2\% en Uruguay. El porcentaje en ese entonces era del 6\% en Canadá; rondaba el 9\% en España, Brasil y Argentina; en Chile representaba el 11,5\% y en Italia y Nueva Zelanda el 14\%. Ya a finales del siglo XX, los países hoy desarrollados presentan ratios que superan el $20 \%$ y, entre los de la región, si bien Uruguay tiene uno de los más altos, se ubica en ese entorno. El dato llama la atención, en la medida en que un inicio tan auspicioso podría haber conducido a un desempeño más dinámico (cuadro A-1).

Para dar cuenta de la dinámica de crecimiento entre gasto público y producto, se realizó un análisis de cointegración de acuerdo al método de Johansen (1991). La cointegración refleja la presencia de un equilibrio de largo 
plazo hacia el cual converge la relación entre ambas variables en el tiempo. Las estimaciones se realizaron utilizando las series de producto y gasto público total y per cápita en términos constantes de Argentina, Brasil, Chile, España, Italia, Canadá, Nueva Zelanda y Uruguay en el período $1900-2000^{3}$.

Las formas de las relaciones de cointegración seleccionadas para testear por este método son las que se aplican con más frecuencia para contrastar la «ley de Wagner», a saber ${ }^{4}$ :

$$
\begin{aligned}
& G=\alpha+\beta Y \\
& \frac{G}{N}=\alpha+\beta \frac{Y}{N}
\end{aligned}
$$

donde $Y=$ PBI; $\mathrm{G}=$ gasto público real del GC; $\frac{Y}{N}=\mathrm{PBI}$ real per cápita; $\frac{G}{N}=$ gasto público real per cápita. Todas las variables se encuentran expresadas en logaritmos. El coeficiente $\beta$ de la relación de cointegración representa una elasticidad de largo plazo, es decir, indica cuánto es la variación porcentual del gasto por cada punto que aumenta el producto. En el apéndice se brindan los detalles del procedimiento utilizado.

Tanto para Uruguay como para los otros países se encontró una relación de largo plazo o de cointegración entre gasto y producto. El cuadro 1 muestra la estimación de los coeficientes $\beta$ de las dos especificaciones estudiadas, junto a la tasa real acumulativa anual de crecimiento del producto total y per cápita:

Para todos los países y en ambos modelos, la elasticidad de largo plazo del gasto público respecto al producto es mayor a la unidad, lo que expresa que los países han ido aumentando la participación del gasto en el producto durante el período.

En términos comparados, Uruguay presenta los coeficientes más bajos (a excepción de Italia en el modelo con variables per cápita). También la expansión real de su producto muestra el menor dinamismo y comparte con Argentina y Nueva Zelanda los crecimientos más reducidos, considerando la población. Es importante destacar el rezago uruguayo respecto al resto de los países considerados, a los que superaba en tamaño del sector público al inicio del período y con los que compartía condiciones económicas relativamente

3 Los datos corresponden al Gobierno Central y al Gobierno Federal, en el caso de Canadá. Para Argentina y Brasil, la información sobre gasto y producto se obtuvo de OXLAD (Oxford Latin American Economic History Database) y de IBGE; en el caso de Chile, el gasto fue tomado de Wagner, Jofré y Lüders (2000) y el producto de Braun et al. (2000). La actualización de las series entre 1995 y 2000 fue provista por José Jofré. Los datos de España se obtuvieron de Carreras y Tafunell (coords.) (2005). Para Italia, entre 1900 y 1975, los datos sobre gasto público se tomaron de Flora y Heidenheimer (eds. 1981) y se completaron con Anuarios de FMI. Para Canadá, la información se obtuvo de la oficina de estadísticas (Statistics Canada). Finalmente, para Nueva Zelanda, se tomaron las series compiladas por el Tesoro (New Zeland Treasury). Los datos sobre población y producto en paridad de poderes de compra corresponden a Maddison (2001).

${ }^{4}$ Las formas funcionales utilizadas se recogen en la revisión realizada en Peacock y Scott (2000) y en Chang (2002), y fueron aplicadas en Comin et al. (2008). 


\section{CUADRO 1}

ELASTICIDAD INGRESO DEL GASTO PÚBLICO DEL GOBIERNO CENTRAL Y TASAS DE CRECIMIENTO DEL PRODUCTO REAL

(En puntos porcentuales y en porcentaje)

\begin{tabular}{|c|c|c|c|c|}
\hline \multirow[b]{2}{*}{ Países } & \multicolumn{2}{|c|}{ Elasticidad de largo plazo $(\beta)$} & \multicolumn{2}{|c|}{ Tasa de crecimiento real $(1900-2000)$} \\
\hline & $\begin{array}{l}\text { Modelo 1: } \\
G=\alpha+\beta Y \\
\end{array}$ & $\begin{array}{c}\text { Modelo 2: } \\
\frac{G}{N}=\alpha+\beta \frac{Y}{N}\end{array}$ & $\mathbf{Y}$ & $\frac{Y}{N}$ \\
\hline Uruguay $^{(*)}$ & 1,09 & 1,17 & 2,6 & 1,4 \\
\hline Argentina $^{(\dagger)}$ & 1,26 & 1,72 & 3,2 & 1,1 \\
\hline Brasil $^{(\dagger)}$ & 1,22 & 1,46 & 4,5 & 2,2 \\
\hline Chile & 1,34 & 1,71 & 3,5 & 1,8 \\
\hline España $^{(\dagger)}$ & 1,45 & 1,61 & 3,0 & 2,2 \\
\hline Italia & 1,15 & 1,15 & 3,0 & 2,4 \\
\hline Canadá & 1,19 & 1,29 & 3,9 & 2,1 \\
\hline Nueva Zelanda & 1,24 & 1,48 & 3,0 & 1,4 \\
\hline
\end{tabular}

Fuentes: Elaborado a partir de datos del apartado 2 (Uruguay) y OXLAD; IBGE; Wagner, Jofré y Lüders (2000); Braun et al. (2000); Carreras y Tafunell (coords.) (2005); Flora y Heidenheimer (eds. 1981); Anuarios de FMI; Statistics Canada; Tesoro- Nueva Zelanda; Maddison (2001) para el resto de los países.

(*) Para Uruguay la serie se inicia en 1903.

(†) La estimación realizada arrojó resultados similares a los presentados en Comín et al. (2008).

similares en ese entonces. Todos estos países, aún transitando diferentes senderos de desarrollo y presentando grados distintos de complejidad en sus estructuras estatales, mostraron una expansión del gasto público del GC más dinámica que la local.

En consecuencia, Uruguay se destaca por el bajo ritmo de crecimiento de su gasto público, que se explica tanto por el lento aumento de esta variable fiscal en relación con la riqueza, como por la modestia de su trayectoria de crecimiento económico de largo plazo. En las razones de este lento ritmo de expansión del gasto podría influir la temprana incidencia directa del sector público en la economía local, reflejada en un nivel elevado del ratio gasto/producto, que luego fue evolucionando de forma moderada.

Las implicancias de esta evolución para el gasto social pueden aproximarse considerando la participación de las partidas de salud y educación en el gasto del GC, como muestra el cuadro $2^{5}$ :

Durante las dos primeras décadas del siglo XX, el gasto en educación y salud se expandió hasta representar casi el 30\% del total. Los años posteriores a

5 El objetivo de contrastar los resultados obtenidos implicó tomar como referencia al Gobierno Central, que comprende la mayor parte del gasto en educación y salud, aunque no incluye la porción más relevante de la seguridad social, que suele registrarse en forma separada. 


\section{CUADRO 2}

PARTICIPACIÓN DEL GASTO PÚBLICO EN EDUCACIÓN Y SALUD EN EL GASTO DEL GOBIERNO CENTRAL URUGUAYO

(En porcentaje)

\begin{tabular}{|l|c|}
\hline Décadas & Gasto en educación y salud/Gasto Gobierno Central (Uruguay) \\
\hline $1910-1920$ & 16,0 \\
\hline $1920-1930$ & 28,8 \\
\hline $1930-1940$ & 23,3 \\
\hline $1940-1950$ & 18,7 \\
\hline $1950-1960$ & 22,7 \\
\hline $1960-1970$ & 31,7 \\
\hline $1970-1980$ & 27,6 \\
\hline $1980-1990$ & 26,0 \\
\hline $1990-2000$ & 29,5 \\
\hline
\end{tabular}

Fuente: Elaborado a partir de datos del apartado 2.

la crisis de 1929, el período de la Segunda Guerra Mundial y su inmediata posguerra implicaron un retroceso en esa ponderación. Luego, aunque con fluctuaciones, desde la década de 1960 se mantuvo en torno al 29\%, incluso tras procesos como el estancamiento y la crisis económica de fines de los sesenta, que fue seguida de la instalación del gobierno dictatorial (de 1973 a 1984), la «crisis de la deuda externa» (tras 1982) y la década de las reformas estructurales de corte liberal, característica de los años noventa en la región ${ }^{6}$.

Como consecuencia, la participación de estas dos partidas de gasto social en términos del producto apenas creció en el período: representaba el $2 \%$ hacia 1910, alcanzó el 4\% en los años veinte y mantuvo ese nivel hasta los años sesenta. Una mirada comparada a la evolución seguida por los otros países aquí considerados desde mediados del siglo XX da cuenta del escaso dinamismo que caracterizó al país (cuadro 3).

La información se presenta dividida en dos períodos: el primero, de auge y consolidación del «estado de bienestar» en los países desarrollados y de «industrialización dirigida por el estado» en los casos latinoamericanos; el segundo, de instalación del nuevo consenso crítico a la intervención del estado en la economía y de reformas estructurales en el manejo de las políticas económicas y sociales que, con variaciones, tuvo lugar en ambos tipos de economía.

6 La «crisis de la deuda externa» se inició en 1982, cuando México anunció la moratoria en el pago de su deuda externa, afectando al acceso al crédito de la mayoría de los países de la región. Para Uruguay, su superación estuvo asociada a sucesivas renegociaciones que -bajo la tutela del Fondo Monetario Internacional- culminaron con el ingreso del país en el Plan Brady (1991). 
CUADRO 3

PARTICIPACIÓN DEL GASTO PÚBLICO EN EDUCACIÓN Y SALUD EN EL PRODUCTO

(En porcentaje)

\begin{tabular}{|l|c|c|c|}
\hline \multirow{2}{*}{ Países } & \multicolumn{2}{|c|}{ Gasto en educación y salud/PBI } & \multirow{2}{*}{ Var. entre períodos } \\
\cline { 2 - 4 } & Promedio (1950-1979) & Promedio (1980-2000) & 40,3 \\
\hline Uruguay & 3,8 & 5,3 & 97,3 \\
\hline Argentina & 3,2 & 6,2 & 174,1 \\
\hline Brasil & 1,8 & 4,8 & 9,2 \\
\hline Chile & 5,3 & 5,8 & 84,9 \\
\hline España & 4,3 & 8,0 & 22,2 \\
\hline Italia & 9,1 & 11,1 & 24,6 \\
\hline Canadá & 9,4 & 11,7 & 49,8 \\
\hline Nueva Zelanda & 7,1 & 10,7 & . \\
\hline
\end{tabular}

Fuentes: Elaborado a partir de datos del apartado 2 (Uruguay); FMI (varios números); Flora y Heidenheimer (eds. 1981); IBGE; Lindert (2004); MECON (2007); Naciones Unidas (varios números); OXLAD; RISALC, Wagner, Jofré y Lüders (2000) para el resto de los países.

Uruguay se encuentra entre los países con menor inversión en educación y salud en ambas etapas. De hecho, si bien Chile presentó el menor dinamismo, desde mediados del siglo XX lo superó en la porción de su riqueza destinada a estas partidas sociales. Más aún: el lugar de relativo privilegio que ocupaba la economía uruguaya en relación con Argentina y Brasil hasta los años ochenta, también tendió a diluirse en los últimos veinte años del período.

La comparación con los otros países muestra un atraso significativo de Uruguay desde el primer subperíodo, incluso considerando que Italia y Canadá recién avanzaron en la implantación de amplios programas sociales luego de la segunda posguerra. En Nueva Zelanda, aunque se mantuvo modesta hasta los años cincuenta, la inversión en partidas sociales se realizó desde las primeras décadas del siglo (Lindert 2004). La evolución seguida por Uruguay también se encuentra lejos del caso español, aun partiendo de un nivel similar. En España, los recursos públicos orientados al gasto social eran residuales en la década de 1950. Los gastos sociales se desarrollaron significativamente a partir de 1979, tras el «Pacto de la Moncloa» con que se cerró el franquismo (Carreras y Tafunell 2005). El crecimiento logrado se encuentra entre los mayores de la muestra.

Los datos permiten reconocer la condición pionera de Uruguay en relación con la temprana incidencia económica del estado y con la inversión en partidas sociales. No obstante, tras un inicio auspicioso la evolución de largo plazo de ambos indicadores ha sido modesta: los recursos capturados por el sector 
público crecieron poco y, en ese marco, el gasto en educación y salud no se amplió. Así, lejos de protagonizar un proceso de convergencia con los países ahora desarrollados, Uruguay fue relegando posiciones durante el período, incluso respecto a los países que realizan un mayor esfuerzo fiscal de la región.

\section{COMPORTAMIENTO CÍCLICO DE LAS VARIABLES FISCALES}

Un manejo contracíclico o neutral al ciclo de las partidas de gasto social, esto es, asegurar que se mantenga elevado (o crezca) en las fases bajas del ciclo y que su volatilidad se reduzca, puede mejorar la efectividad de los programas de protección social. Sin embargo, para los países de la región, la evidencia para las últimas décadas del siglo XX ha mostrado prociclicidad en las partidas de educación y salud y resultados variados para seguridad social.

En el presente apartado se explora si el gasto público total y social en Uruguay ha logrado protegerse de las fluctuaciones de la economía, considerando el período 1910-2000.

La volatilidad de las partidas fiscales y del producto se calculó tomando los desvíos estándar del componente cíclico de las series (estimado aplicando el filtro de Hodrick-Prescott, Hodrick y Prescott, 1997) como proporción de su tendencia. El comportamiento de las series en el ciclo se analizó a través de correlaciones entre los componentes cíclicos de las partidas fiscales y del producto, de acuerdo a Talvi y Vegh (2000) y Mailhos y Sosa (2000). El foco en los componentes cíclicos permite eliminar las correlaciones derivadas de los incrementos tendenciales de las variables en el largo plazo. Una correlación positiva y significativa indica un comportamiento procíclico; una correlación negativa y significativa revela contraciclicidad. Si la correlación no es significativamente diferente de cero, no puede rechazarse un comportamiento neutro al ciclo. Las estimaciones se muestran en el cuadro 4.

El desvío estándar de las variables fiscales fue muy superior al del producto. Ello resulta significativo, ya que diversos estudios han marcado la elevada volatilidad cíclica del PBI de Uruguay (Kamil y Lorenzo 1998, Bértola y Lorenzo 2004, Cal 2004 y Carbajal y De Melo 2007). La volatilidad fue especialmente elevada para los gastos en educación, salud y seguridad social. Estos resultados implican una fuerte vulnerabilidad del gasto público y de sus componentes a los cambios en el entorno macroeconómico, reflejando que, en el largo plazo, las variables fiscales no parecen haber jugado un rol estabilizador, sino todo lo contrario.

El cálculo de las correlaciones arrojó valores positivos y significativos para todas las variables fiscales, salvo para la seguridad social. Lo cual indica un manejo procíclico del gasto total, en educación y en salud. Para la seguridad social el dato señala la existencia de un comportamiento neutral al ciclo. Este desempeño podría encubrir dinámicas diferentes en el ciclo de los pagos de pasividades y las prestaciones a activos. 
CUADRO 4

DESVÍO ESTÁNDAR Y CORRELACIÓN ENTRE COMPONENTES CÍCLICOS DE LAS VARIABLES FISCALES Y DEL PRODUCTO, 1910-2000

\begin{tabular}{|l|l|c|c|l|l|c|}
\hline & \multicolumn{6}{|c|}{ Componentes cíclicos, 1910-2000 } \\
\cline { 2 - 7 } $\begin{array}{l}\text { Coeficientes } \\
\text { estimados }\end{array}$ & PBI & $\begin{array}{c}\text { Gasto Gobierno } \\
\text { Central Con- } \\
\text { solidado }\end{array}$ & $\begin{array}{c}\text { Gasto } \\
\text { Gobierno } \\
\text { Central }\end{array}$ & $\begin{array}{c}\text { Gasto en } \\
\text { educación }\end{array}$ & $\begin{array}{c}\text { Gasto en } \\
\text { salud }\end{array}$ & $\begin{array}{c}\text { Gasto en } \\
\text { seguridad } \\
\text { social }\end{array}$ \\
\hline $\begin{array}{c}\text { Desvío } \\
\text { estándar }\end{array}$ & 0,04 & 0,08 & 0,08 & 0,1 & 0,12 & 0,13 \\
\hline $\begin{array}{c}\text { Correlación } \\
\text { con el PBI }\end{array}$ & 1 & $0,3937 * * *$ & $0,351 * * *$ & $0,3564 * * *$ & $0,3016^{* * *}$ & 0,0399 \\
\hline P-Valor & & 0,0001 & 0,0006 & 0,0005 & 0,0037 & 0,7074 \\
\hline
\end{tabular}

*** Significativo al 1\%. Para las estimaciones se utilizó el filtro de Hodrik y Prescott (1997) con un parámetro de suavizado de 6,25, como recomiendan Ravn y Uhlig (2002) para datos anuales. Las conclusiones se mantienen con un parámetro de suavizado de 100, como sugerían Hodrik y Prescott.

Para los países utilizados como referencia en el apartado 1, también se calculó el comportamiento cíclico del gasto total. Los resultados fueron heterogéneos. Mientras España, Brasil y Canadá presentaron un perfil neutro en el largo plazo, Argentina, Nueva Zelanda, Chile e Italia fueron procíclicos, incluso estos dos últimos en mayor medida que Uruguay (cuadro A-2). Sin embargo, mientras Italia desde 1950 y Chile a partir de 1980 presentaron neutralidad cíclica, Uruguay mostró niveles crecientes de prociclicidad que se acentuaron hacia finales del período (Azar y Fleitas 2010).

En síntesis, en el largo plazo el gasto público uruguayo presentó un comportamiento volátil y procíclico, coincidiendo con lo hallado en otros estudios para el corto o mediano plazo. Estos resultados se encuentran en línea con lo planteado en Oddone (2005), donde se fundamenta que, desde mediados de siglo, las políticas macroeconómicas, lejos de contribuir a suavizar los ciclos en el país, los han profundizado, generando así una penalización al crecimiento. El sesgo procíclico del gasto en su conjunto se reiteró para educación y salud, con probables consecuencias adversas en términos de eficiencia y del logro de bienestar. Finalmente, la seguridad social tuvo un comportamiento neutro, si bien fue la partida con mayor volatilidad agregada.

\section{GASTO PÚBLICO SOCIAL, AJUSTE Y DESAJUSTE FISCAL}

Un aspecto adicional para evaluar la importancia otorgada a la preservación del gasto social durante el período, se vincula a su manejo en situaciones en que fue necesario resolver desequilibrios en las cuentas públicas. Siguiendo la metodología de Cominetti (1994), se buscó distinguir 
qué comportamiento tuvieron los gastos en educación, salud y seguridad social en momentos de desequilibrios presupuestales.

A partir de los datos de resultado fiscal del Gobierno Central en relación al PBI, se establecieron fases de «desajuste fiscal» y de «ajuste fiscal». Las primeras refieren a años consecutivos de aumento de las necesidades de financiamiento y, por tanto, de deterioro de las finanzas públicas, y las segundas a años consecutivos de caída, es decir, de ajustes fiscales.

El diagrama de restricciones fiscales mostró que los mejores resultados correspondieron a los primeros años del siglo XX (en los cuales se lograron mayoritariamente superávits). En cambio, desde la década de 1970 los mejores resultados fueron déficit de más de un punto porcentual del producto y se encontró más apretada la secuencia de fases de desajustes. En los años intermedios, si bien los ratios no llegaron a ser globalmente positivos, fueron moderados en comparación con los del último cuarto de siglo.

Cabe aclarar que, definidas de esta forma, las fases no necesariamente corresponden a los diferentes momentos del ciclo económico. En este sentido, las fases de ajuste o desajuste se asocian al componente discrecional de la política, ya que además de recoger los efectos cíclicos de la recaudación y el gasto, incluyen las decisiones presupuestales sobre el resultado fiscal.

Dado el conjunto de fases de ajuste y desajuste encontradas a lo largo del siglo se indagó en la contribución de los diferentes gastos sociales a los desequilibrios. De primar el rol de protección social típico de estos gastos, es esperable su mantenimiento a costa del ajuste de otras partidas. Si, por el contrario, se utilizaron como instrumento del ajuste, se habrían deteriorado igual que el resto de las finanzas públicas.

A efectos de dar cuenta de la variación del ratio gasto social/PBI en cada una de las fases identificadas, se compararon las variaciones del gasto social y del producto. Se reveló el número de veces en que las partidas de gasto social fueron "pro ajuste», actuando para reducir los desequilibrios en momentos de ajuste fiscal o "pro desajuste», cuando dificultaron esa reducción. También se revisó el número de casos de desequilibrio fiscal en que los gastos sociales tendieron a agravarlo (fueron "pro desajuste») o contribuyeron a amortiguarlo (fueron "pro ajuste») ${ }^{7}$.

En el cuadro 5 se resume el número de casos en que el comportamiento de las partidas de gasto social fue «pro ajuste» o «pro desajuste» durante el siglo. En el cuadro A-3 se muestra el porcentaje de ajuste y desajuste de cada partida, por fase.

El énfasis de la información presentada reside en la dirección del efecto. Como puede observarse, la tendencia a utilizar el gasto en educación para

\footnotetext{
7 De acuerdo a la definición propuesta por Cominetti, un sesgo "pro ajuste» representa una reducción del gasto social o un aumento que es proporcionalmente menor al que experimenta el producto. En cambio, un sesgo "pro desajuste» implica un aumento del gasto social o una disminución menor a la del producto.
} 


\section{CUADRO 5}

NÚMERO DE EPISODIOS PRO AJUSTE O PRO DESAJUSTE DE LAS PARTIDAS DE GASTO SOCIAL POR FASE

\begin{tabular}{|c|c|c|c|c|}
\hline Fases & Comportamiento & $\begin{array}{c}\text { Gasto en } \\
\text { educación }\end{array}$ & $\begin{array}{l}\text { Gasto en } \\
\text { salud }\end{array}$ & $\begin{array}{c}\text { Gasto en seguridad } \\
\text { social }\end{array}$ \\
\hline \multirow{2}{*}{$\begin{array}{l}\text { Ajuste (13 epi- } \\
\text { sodios) }\end{array}$} & pro ajuste & 9 & 5 & 7 \\
\hline & pro desajuste & 4 & 8 & 6 \\
\hline \multirow{2}{*}{$\begin{array}{l}\text { Desajuste (11 epi- } \\
\text { sodios) }\end{array}$} & pro ajuste & 6 & 3 & 1 \\
\hline & pro desajuste & 5 & 8 & 10 \\
\hline
\end{tabular}

Fuentes: Elaboración propia a partir de datos de Azar et al. (2009).

amortiguar desequilibrios en las fases de ajuste fue más definida que la de salud y seguridad social. Ello se refleja en el carácter "pro ajuste» del primer gasto en los episodios de restricción fiscal.

Del otro lado, los gastos en salud y seguridad social presentaron mayor flexibilidad para expandirse al ritmo de los desequilibrios fiscales. Por tanto, frecuentemente contribuyeron a la situación de desajuste fiscal. Nuevamente, se encuentra que el gasto en educación tuvo un manejo mucho menos expansivo en estas circunstancias y mantuvo una orientación "pro ajuste» aún en fases de desajuste.

Aunque la evidencia reunida no permite realizar afirmaciones concluyentes, vale notar que el manejo del gasto en educación ha sido diferente respecto al resto de los gastos sociales considerados. Esta partida parece haber contribuido más al ajuste y haberse beneficiado menos del desajuste que el resto de los gastos.

\section{SOBRE LA EXISTENCIA DE TRES REGÍMENES FISCALES}

La propuesta de este apartado consiste en utilizar las metodologías ya aplicadas para testear si pueden encontrarse diferencias entre etapas asociadas a «regímenes fiscales». Tal como se plantea en Azar et al. (2009), los regímenes se identificaron en función de la utilización de indicadores sobre tamaño del estado, necesidades de financiamiento y mecanismos para cubrirlo; rol redistributivo del sector público y un relevamiento de ensayos y reformas de las leyes presupuestales, de la estructura tributaria y de la orientación del gasto público. Como los aspectos considerados en este artículo no fueron discutidos en la caracterización precedente, se indaga si la evidencia aquí reunida contribuye a la caracterización de estos regímenes o si, por el contrario, genera una clasificación distinta de las etapas históricas. 
En términos generales, los regímenes fiscales descriptos en Azar et al. (2009) están asociados con las clásicas fases de desarrollo económico que transitaron los países de América Latina después de consolidados los estados independientes. Así, el primer régimen transcurre con el modelo agroexportador, que se extendió desde fines del siglo XIX hasta la Gran Depresión; el segundo, con la industrialización dirigida por el Estado (que comenzó a gestarse en los años treinta y que, en Uruguay, llegó hasta la década de 1960) y el tercero, con la aplicación de políticas económicas de corte liberal, que promovieron la apertura externa y un rol más amplio del mercado, que caracterizó al último cuarto del siglo XX.

En el capítulo I de la obra, los autores destinan un apartado a la descripción somera de los rasgos distintivos de los regímenes. Allí señalan que el primero estuvo caracterizado por una concepción de las finanzas públicas «ortodoxa», cuyo objetivo principal era un manejo fiscal neutral, preservado a costa de no avanzar decididamente en metas redistributivas. Durante el segundo régimen, la actuación gubernamental buscó promover la protección social e implantó políticas distributivas, auspiciando además el proceso de acumulación en la industria. Se destaca la universalización de la cobertura de la seguridad y la asistencia social, la expansión de los servicios de enseñanza (especialmente media), la provisión estatal de agua potable y saneamiento y el peso significativo de las subvenciones y subsidios para fomentar sectores de actividad y fijar precios máximos a los bienes de la canasta básica ${ }^{8}$. La búsqueda de equilibrios fiscales quedó subordinada a la promoción del «crecimiento hacia dentro». El tercer régimen fiscal se caracteriza por la disciplina fiscal (relevante además para obtener crédito externo) y la aplicación de políticas de asistencia social focalizadas como características clave.

Tomando como cierta la construcción de los regímenes, los indicadores presentados en los apartados anteriores se estimaron para los siguientes subperíodos: $1905-1931 ; 1932-1971$ y $1972-2000^{9}$.

Dado que los intervalos de los regímenes fiscales son relativamente cortos, la estimación de las elasticidades se realizó con la metodología de Engle y Granger (1987). Según ésta, primero debe estimarse la relación de cointegración por Mínimos Cuadrados Ordinarios (MCO) y después testearse el orden de integración de los residuos de esa estimación. Si las series son I(1) y los residuos de la relación son $\mathrm{I}(0)$ existe cointegración y los coeficientes estimados por MCO representarán las elasticidades de largo plazo. Para testear el orden de integración de las series se utiliza el test Dickey-Fuller Aumentado (ADF);

8 Entre las regulaciones más destacadas puede señalarse la implementación de regulaciones salariales, la fijación de salarios mínimos y las asignaciones familiares (1943); la creación de la Comisión de Subsistencias (1947), y el control de precios de alquileres (1948).

9 Si bien en Azar et al. (2009) los años que sirven de límite para definir los regímenes son 1930 y 1973, en este trabajo, y a fin de realizar los cálculos, se tomaron como límite los años 1931 y 1971. La decisión se fundamenta en que en estos años el componente cíclico del producto es cercano a cero, lo que evita dificultades provenientes de las fluctuaciones económicas a la hora de comparar elasticidades. 


\section{CUADRO 6 \\ ELASTICIDAD INGRESO DEL GASTO DEL GOBIERNO CENTRAL POR REGÍMENES FISCALES \\ (Puntos porcentuales)}

\begin{tabular}{|l|c|c|c|c|}
\hline & $\mathbf{1 9 0 5 - 2 0 0 0}$ & $\begin{array}{c}\mathbf{1 .}^{\mathbf{e r}} \text { régimen } \\
\text { fiscal }\end{array}$ & $\begin{array}{c}\mathbf{2}^{\circ} \text { régimen fis- } \\
\text { cal }\end{array}$ & $\begin{array}{c}\mathbf{3}^{\text {er }} \\
\text { régimen } \\
\text { fiscal }\end{array}$ \\
\hline $\begin{array}{c}\text { Elasticidad Modelo I } \\
\text { LnG }=\alpha+\beta \ln Y\end{array}$ & 1,060 & 0,609 & 0,824 & 1,183 \\
\hline Valor ADF Modelo I & $-2,819 * * *$ & $-2,547^{* * *}$ & $-2,007^{* *}$ & $-1,737^{*}$ \\
\hline $\begin{array}{c}\text { Elasticidad Modelo II } \\
\text { Ln } \frac{G}{N}=\alpha+\beta \ln \frac{Y}{N}\end{array}$ & 1,098 & 0,435 & 0,729 & 1,231 \\
\hline Valor ADF Modelo II & $-2,815^{* * *}$ & $-2,292^{* *}$ & $-2,035^{* * *}$ & $-1,758^{*}$ \\
\hline Datos para ADF & 94 & 25 & 36 & 25 \\
\hline Valor McKinnon 1\% & $-2,590$ & $-2,661$ & $-2,631$ & $-2,661$ \\
\hline Valor McKinnon $5 \%$ & $-1,944$ & $-1,955$ & $-1,950$ & $-1,955$ \\
\hline Valor McKinnon 10\% & $-1,614$ & $-1,609$ & $-1,611$ & $-1,609$ \\
\hline
\end{tabular}

Nota: se rechaza la hipótesis nula de raíz unitaria al $10 \%(*)$, al $5 \%(* *)$ y al $1 \%(* * *)$.

pero dado el supuesto de cointegración, los valores críticos del mismo deben ser corregidos utilizando MacKinnon (2010).

Las series presentaron raíces unitarias en sus niveles (series I(1)). Luego se estimaron las relaciones de cointegración y se realizó el test $\mathrm{ADF}$, con los valores críticos corregidos. Los resultados obtenidos se presentan en el cuadro 6.

Como forma de verificar la robustez de los resultados se estimó la relación de cointegración para todo el período, como se realizara a partir de Johansen (1991). Al igual que entonces, se encontró una relación de cointegración en el largo plazo significativa al 1\%, siendo el coeficiente apenas mayor a la unidad. Para el primer y el segundo regímenes, en cualquiera de los modelos utilizados, se obtuvo evidencia de cointegración al 1\% de significación. En este marco, las elasticidades de largo plazo no difieren significativamente entre el primer y el segundo régimen fiscal, aunque ambas son menores a la unidad, señalando el bajo crecimiento del gasto en relación al producto en este período. En cuanto al tercer régimen, la evidencia a favor de la cointegración sólo puede sostenerse al 10\% de confianza. Los coeficientes para este régimen son mayores que uno, con lo que la elasticidad parecería haber sido creciente hacia el final del período.

La volatilidad y los coeficientes de correlación entre los componentes cíclicos de las variables fiscales y el producto por régimen fiscal se presentan en el cuadro 7:

Los datos muestran una acentuación del patrón procíclico del gasto total y sobre todo del gasto en educación y salud a través de las etapas, acompañado 
CUARDRO 7

DESVÍO ESTÁNDAR Y CORRELACIÓN ENTRE COMPONENTES CÍCLICOS DE LAS VARIABLES FISCALES Y DEL PRODUCTO POR RÉGIMEN FISCAL

\begin{tabular}{|c|c|c|c|c|c|c|}
\hline \multicolumn{7}{|c|}{ Componente cíclicos } \\
\hline \multirow[t]{2}{*}{$\begin{array}{l}\text { Coeficientes } \\
\text { estimados }\end{array}$} & PBI & $\begin{array}{l}\text { Gasto Gobierno } \\
\text { Central Con- } \\
\text { solidado }\end{array}$ & $\begin{array}{l}\text { Gasto } \\
\text { Gobierno } \\
\text { Central }\end{array}$ & $\begin{array}{c}\text { Gasto en } \\
\text { educación }\end{array}$ & $\begin{array}{l}\text { Gasto en } \\
\text { salud }\end{array}$ & $\begin{array}{c}\text { Gasto en } \\
\text { seguridad } \\
\text { social }\end{array}$ \\
\hline & \multicolumn{6}{|c|}{ Primer régimen: 1910-1931 } \\
\hline $\begin{array}{l}\text { Desvío } \\
\text { estándar }\end{array}$ & 0,05 & 0,06 & 0,06 & 0,06 & 0,11 & 0,16 \\
\hline $\begin{array}{c}\text { Correlación } \\
\text { con el PBI }\end{array}$ & 1 & 0,298 & 0,3042 & 0,3176 & 0,3305 & $-0,904$ \\
\hline \multirow[t]{2}{*}{ P-Valor } & & 0,1780 & 0,1687 & 0,1498 & 0,1330 & 0,6891 \\
\hline & \multicolumn{6}{|c|}{ Segundo régimen: 1932-1971 } \\
\hline $\begin{array}{l}\text { Desvío } \\
\text { estándar }\end{array}$ & 0,03 & 0,1 & 0,1 & 0,11 & 0,12 & 0,14 \\
\hline $\begin{array}{c}\text { Correlación } \\
\text { con el PBI }\end{array}$ & 1 & $0,3960 * *$ & $0,4006 * *$ & $0,2985^{*}$ & 0,2462 & 0,1851 \\
\hline \multirow[t]{2}{*}{ P-Valor } & & 0,0114 & 0,0104 & 0,0613 & 0,1257 & 0,2528 \\
\hline & \multicolumn{6}{|c|}{ Tercer régimen: 1972-2000 } \\
\hline $\begin{array}{l}\text { Desvío } \\
\text { estándar }\end{array}$ & 0,03 & 0,08 & 0,08 & 0,11 & 0,12 & 0,1 \\
\hline $\begin{array}{c}\text { Correlación } \\
\text { con el PBI }\end{array}$ & 1 & $0,6006 * * *$ & $0,3925 * *$ & $0,5983 * * *$ & $0,3802 * *$ & $-0,0149$ \\
\hline P-Valor & & 0,0006 & 0,0352 & 0,0006 & 0,0419 & 0,939 \\
\hline
\end{tabular}

Nota: nivel de significación expresado para 10\% (*), 5\%(**) y 1\%(**). Para las estimaciones se utilizó el filtro de Hodrik y Prescott (1997) con un parámetro de suavizado de 6,25 tal como recomiendan Ravn y Uhlig (2002) para datos anuales, De cualquier manera las conclusiones se mantienen cuando se utiliza el parámetro de suavizado igual a 100 tal como recomendaban Hodrik y Prescott.

por una amortiguación de la volatilidad en todas las variables hacia finales del período. En general, las variables fiscales presentan una mayor variabilidad que el producto, especialmente las partidas de gasto social.

En el primer régimen fiscal, la volatilidad del producto fue la más elevada. Probablemente, esto se vincule con los fuertes shocks a los que estuvo sometida la economía durante esos años: una crisis financiera local en 1913; la Primera Guerra Mundial; la expansión de los años veinte y los primeros efectos de la Gran Depresión. Sin embargo, los gastos en educación y salud en las primeras décadas del siglo mostraron las menores fluctuaciones. 
Por su parte, ninguna de las partidas analizadas exhibió un patrón cíclico definido, lo que apuntaría a la neutralidad cíclica de las variables, en línea con un manejo prudente de las finanzas públicas. Este comportamiento difiere marcadamente de lo hallado para las otras dos etapas.

Para los años que identifican al segundo régimen fiscal, la volatilidad del producto fue menor que la estimada para el período previo. Este hallazgo parece factible en una etapa caracterizada por el cerramiento de la economía y el impulso del «crecimiento hacia dentro». Los gastos sociales, sin embargo, presentaron una volatilidad alta. El patrón de comportamiento procíclico se verificó tan sólo para los gastos totales y muy débilmente para el gasto en educación. Este dato coincide con una mayor discrecionalidad en el manejo de los gastos en este período, ya apuntado en Oddone (2005). Como resultado, parece haberse mantenido bajo relativo resguardo el gasto en salud y seguridad social, y, en menor medida, en educación, todos muy caros para el modelo de crecimiento basado en el fortalecimiento del mercado interno.

En el último régimen, la volatilidad del producto fue la menor de las halladas, aún cuando incluye la "crisis de la deuda» con que se abrió la década de 1980, que implicó una fuerte caída del nivel de actividad (del orden del 15\%). En la misma línea, los gastos totales y sociales presentaron una variabilidad menor respecto al período anterior, dando indicios de un manejo fiscal más estable que en el pasado. Sin embargo, la menor volatilidad del producto no fue acompañada de un perfil más neutro de la política fiscal, que, por el contrario, presentó un comportamiento marcadamente procíclico en relación con las etapas anteriores. El mismo resultado fue hallado en Stein, Talvi y Grisanti (1998) y Mailhos y Sosa (2000). Por su parte, la seguridad social mantuvo un perfil neutro en el período, aun cuando experimentó un importante crecimiento (gráfico 2).

Finalmente, se investigó el comportamiento del gasto social en las etapas de ajuste y desajuste fiscal por régimen fiscal, siguiendo la definición utilizada en el apartado 5. En el cuadro 8 se resumen los hallazgos sobre este aspecto:

La información no describe un sesgo definido «pro ajuste» o "pro desajuste» de los gastos sociales que sea propio de cada uno de los diferentes regímenes.

Durante el primer y segundo regímenes, los gastos sociales se restringieron para equilibrar las cuentas públicas en los momentos de ajuste, si bien el gasto en salud fue más difícil de controlar en el primer régimen. En las épocas de ampliación del desequilibrio fiscal, las partidas sociales contribuyeron al desajuste general (en particular, salud y seguridad social). En este sentido, los gastos sociales no parecen haber sido objeto particular de protección frente a las vicisitudes de la gestión financiera en ninguno de los dos regímenes: se ampliaron cuando hubo margen y se restringieron en caso contrario.

El comportamiento en el último régimen fiscal del siglo presentó algunas diferencias con los anteriores. Allí, los gastos en salud y seguridad social tendieron a ser "pro desajuste» en ambas fases. Sin embargo, el gasto en educación actuó en sentido opuesto: tendió a amortiguar desequilibrios en la 
TABLE 8

NÚMERO DE EPISODIOS PRO AJUSTE O PRO DESAJUSTE DE LAS PARTIDAS DE GASTO POR FASE, SEGÚN RÉGIMEN FISCAL

\begin{tabular}{|c|c|c|c|c|}
\hline Fases & Comportamiento & $\begin{array}{c}\text { Gasto en } \\
\text { educación }\end{array}$ & $\begin{array}{c}\text { Gasto en } \\
\text { salud }\end{array}$ & $\begin{array}{c}\text { Gasto en seguridad } \\
\text { social }\end{array}$ \\
\hline \multirow{3}{*}{$\begin{array}{l}\text { Ajuste (4 epi- } \\
\text { sodios) }\end{array}$} & \multicolumn{4}{|c|}{ Primer régimen: 1910-1931 } \\
\hline & pro ajuste & 3 & 1 & 3 \\
\hline & pro desajuste & 1 & 3 & 1 \\
\hline \multirow{2}{*}{$\begin{array}{l}\text { Desajuste ( } 3 \text { epi- } \\
\text { sodios) }\end{array}$} & pro ajuste & 0 & 1 & 0 \\
\hline & pro desajuste & 3 & 2 & 3 \\
\hline \multirow{3}{*}{$\begin{array}{l}\text { Ajuste (4 epi- } \\
\text { sodios) }\end{array}$} & \multicolumn{4}{|c|}{ Segundo régimen: 1932-1971 } \\
\hline & pro ajuste & 4 & 3 & 2 \\
\hline & pro desajuste & 0 & 1 & 2 \\
\hline \multirow{2}{*}{$\begin{array}{l}\text { Desajuste (3 epi- } \\
\text { sodios) }\end{array}$} & pro ajuste & 2 & 1 & 1 \\
\hline & pro desajuste & 1 & 2 & 2 \\
\hline \multirow{3}{*}{$\begin{array}{l}\text { Ajuste (5 epi- } \\
\text { sodios) }\end{array}$} & \multicolumn{4}{|c|}{ Tercer régimen: $1972-2000$} \\
\hline & pro ajuste & 2 & 1 & 2 \\
\hline & pro desajuste & 3 & 4 & 3 \\
\hline \multirow{2}{*}{$\begin{array}{l}\text { Desajuste (5 epi- } \\
\text { sodios) }\end{array}$} & pro ajuste & 4 & 1 & 0 \\
\hline & pro desajuste & 1 & 4 & 5 \\
\hline
\end{tabular}

mayoría de los episodios. Por tanto, salvo por esta orientación del gasto en educación, el manejo del gasto social en el régimen liberal no parece recoger una reducción deliberada del papel del estado en la protección social, en línea con la prédica pro mercado que caracterizó al período.

Sin desconocer las limitaciones de la evidencia presentada, este apartado agrega nueva información para discutir la identificación de los regímenes fiscales durante el siglo XX. Como puede apreciarse, los resultados en los aspectos revisados, no necesariamente coinciden con los esquemas esperados. Así, los datos obtenidos para el primer régimen fiscal parecen ir en línea con una gestión conservadora de las finanzas públicas. En cambio, en el 
segundo régimen, aunque se mantuvo el patrón relativamente acíclico del gasto social, éste presentó una elevada volatilidad y no se preservó deliberadamente frente a los desequilibrios fiscales. Llama la atención esta orientación en el marco de un régimen tradicionalmente concebido como de expansión de la protección social, no sujeta a restricciones de financiamiento (Azar et al. 2009). Finalmente, para el tercer régimen, cuyas características estarían más en línea con la disciplina fiscal, el aumento del gasto público en relación al producto mostró el mayor nivel y el patrón pro-cíclico de los gastos fue el más acentuado. Además, el gasto público social se expandió más allá de lo que podía preverse (se encontró que fue generalmente "pro desajuste», salvo en el caso de educación).

\section{CONSIDERACIONES FINALES}

Los resultados del análisis realizado contribuyen a visibilizar algunos aspectos del rol del gasto público en el desarrollo de la matriz de protección social estatal que caracterizó a las políticas económicas de Uruguay durante el siglo XX.

El trabajo permitió verificar que el modesto incremento del gasto público en el largo plazo se debió tanto al escaso dinamismo del producto como al lento ritmo de expansión - en términos relativos- del propio gasto, por cada punto porcentual de riqueza que el país incrementaba. Como resultado, Uruguay, que fue pionero en términos del nivel de gasto de su Gobierno Central y de la provisión de servicios sociales a principios de siglo, finalizó el período con niveles de gasto similares a los de los países latinoamericanos y menores que los países desarrollados. Este hecho se constata a partir de la comparación con otras economías de la región de alta inversión en gasto social (Argentina, Chile, Costa Rica); con economías europeas de niveles de ingresos similares al comienzo del período (España e Italia) y con economías de «nuevo asentamiento» (Canadá y Nueva Zelanda). En particular, el gasto en educación y salud como porcentaje del producto, que partió de niveles cercanos e incluso mayores al del resto de las economías, mostró una expansión lenta, que implicó que Uruguay perdiera posiciones a lo largo del siglo.

En línea con las tendencias encontradas para los países de América Latina en períodos recientes, cuando se considera durante todo el siglo XX el gasto público del Gobierno Central, el consolidado (es decir, el que incluye la seguridad social) y las partidas de educación y salud han sido significativamente procíclicas. Para el gasto en seguridad social, básicamente compuesto por retribuciones a pasivos, el resultado fue la neutralidad. Por su parte, la volatilidad de las partidas fiscales tendió a superar ampliamente la del producto. En este sentido, el gasto público, e incluso el gasto en educación y salud, lejos de contribuir a amortiguar los ciclos y las fluctuaciones de la economía, habría tendido a profundizarlos. El manejo fiscal parece haber 
conducido a que en las fases bajas del ciclo no se dispusiera de mecanismos para atender las demandas emergentes de las recesiones y crisis y que, en los períodos de alza, se cubrieran los vacíos surgidos en las etapas anteriores sin realizar reservas para futuras épocas de escasez.

Considerando las fases de ajuste y desajuste fiscal, no fue posible detectar una tendencia definida a la preservación del gasto en salud y seguridad social: se ajustaron durante períodos de restricciones fiscales y tendieron a ampliarse en períodos de mayor flexibilidad. Debe destacarse que el gasto en educación pareció utilizarse en mayor medida para amortiguar desequilibrios y, complementariamente, fue menos favorecido en etapas de desajuste fiscal. Presentó, además, la prociclicidad más elevada. Sin duda, este comportamiento tiene consecuencias importantes sobre su calidad, en la medida en que recibió más o menos recursos de acuerdo a la etapa del ciclo en la que se encontraba la economía. En este sentido, el rol de protección y promoción social de este gasto pareció entrar en conflicto con su papel en el ajuste del déficit fiscal con mayor frecuencia que en el resto de los casos.

La exploración del comportamiento de las variables fiscales a largo y corto plazo, y frente a restricciones de financiamiento en los tres regímenes fiscales encontrados para el siglo XX, no permitió identificar diferencias marcadas entre ellos.

Así, la elasticidad del gasto público respecto al producto fue la menor en el primer régimen, creció con el segundo y alcanzó el mayor ratio con el tercero. También la prociclicidad de las partidas fiscales (con excepción de la neutralidad hallada para la seguridad social) fue profundizándose desde el principio hasta el final del período. Este comportamiento llama la atención, ya que podría haberse esperado que en un régimen más comprometido con la responsabilidad fiscal y la búsqueda de equilibrios como el tercero, la elasticidad del gasto y la prociclicidad del gasto se hubieran amortiguado.

En cuanto a la gestión del gasto social, éste tendió a ajustarse a las necesidades de financiamiento del gobierno en el primer y segundo regímenes, en tanto pareció desvincularse de esta restricción y expandirse más definidamente durante el tercer régimen, salvo en el caso del gasto en educación. Como consecuencia, no se encontró la deliberada protección del gasto social frente a los vaivenes de la economía que podía preverse asociada al segundo régimen fiscal. De hecho, esta tendencia pareció reflejarse mejor en el último régimen. En éste, pese a la necesidad de lograr la sostenibilidad de las cuentas públicas y bajo una visión de reducción del rol del estado en la economía, los gobiernos debieron continuar cumpliendo con las funciones acumuladas en el pasado y con las demandas sociales dirigidas al sector público.

En síntesis, se encuentra que las restricciones en la gestión macroeconómica asociadas a las finanzas públicas afectaron instrumentos directamente vinculados a la producción de bienestar. Pese al carácter pionero de Uruguay en materia de protección social a principios de siglo, la lenta 
expansión del gasto público, la volatilidad y prociclicidad de las partidas del gasto social, así como su rol en la estabilización de las cuentas públicas podrían constituir una parte importante de la explicación del rezago experimentado por el país, en relación con otras economías.

\section{FUENTES}

Acevedo, E. (1934, 1965, 1936): Anales Históricos del Uruguay. Montevideo: Barreiro y Ramos.

Banco Central Del Uruguay (BCU): Boletines Estadísticos (varios volúmenes). Montevideo: BCU.

Banco De La República (BROU) (1965): Cuentas Nacionales. Montevideo: Departamento de Investigaciones Económicas del BROU.

Bertino, M. y Tajam, H. (1999): El PBI uruguayo. Montevideo: Facultad de Ciencias Económicas y de Administración (UDELAR)-Comisión Sectorial de Investigación Científica.

Bértola, L.; Calicchio, L.; Camou, M. y Porcile, G. (1999): «Southern cone real wage compared: a purchasing power parity approach to convergence and divergence trends, 1870-1996». Serie Documentos de Trabajo 44, Unidad Multidisciplinaria. Montevideo: Facultad de Ciencias Sociales.

Braun, J.; Braun, M.; Briones, I.; Díaz, J.; Lüders, R. y Wagner, G. (2000): «Economía chilena 1810-1995. Estadísticas Históricas». Documento de Trabajo 187. Santiago de Chile: Pontificia Universidad de Chile.

Carreras, A. y TAfunell, X. (coords.) (2005): Estadísticas Históricas de España. Bilbao: BBVA.

Flora, P. y Heidenheimer, J.A. (eds) (1981): The Development of Welfare States in Europe and America. New Brunswick: Transaction Publishers.

Contaduría General De La Nación (CGN) (1996): «Estudio de la Evolución Presupuestal 1961-1994». Montevideo: CGN.

Corbo, D.; Menéndez, W. y Peri, A. (1989): La evolución del gasto público en educación en el Uruguay en el período 1961-1988. Montevideo: Centro de Estudios para la Democracia Uruguaya.

Fondo Monetario InTERnacional (FMI) (varios volúmenes): Government Financial Statitstics Yearbook. Washington: FMI.

Instituto Brasileiro De Geografía Y Estadística (IBGE): «Estadísticas del siglo XX», en sitio web http://www.ibge.gov.br/seculoxx/.

Instituto Nacional De Estadística (INE): sitio web http://www.ine.gub.uy.

Maddison, A. (2001): «Historical Statistics of the World Economy: 1-2008 $\mathrm{AD}$ ». Groningen: Groningen Growth and Development Centre en sitio: web http://www.ggdc.net.

Ministerio De Economía Y Producción (MECON) (2007): «Presupuesto de la Administración Nacional. Gastos por finalidad, función y naturaleza económica, 1965-2006». Argentina: Oficina Nacional de Presupuesto. 
Ministerio De Salud Pública-Organización Mundial De La Salud (1972): Estudio del gasto en salud 1967-1971. División de Planificación de Salud. Montevideo: Ministerio de Salud Pública.

Naciones Unidas (varios números): Anuarios Estadísticos de Naciones Unidas. New York: Departamento de Asuntos Económicos y Sociales.

Nahum, B. (2006): Estadísticas Históricas del Uruguay, 1900-1950. Montevideo: Departamento de Publicaciones de la Universidad de la República.

New Zeland Treasury: sitio web http://www.treasury.govt.nz/government/data.

Oxford Latin American Economic History Database (OXLAD): sitio web http:// Www oxlad.qeh.ox.ac.uk.

STATISTics CANADA: «Historical Statistics of Canada», sitio web http://www. statcan.gc.ca.

Red De la División De Desarrollo Social De la Cepal (RISAlC): sitio web http://www.risalc.org/portal/.

WAGner, G.; Jofré, J. y LüDERS, R. (2000): «Economía Chilena 1810-1995. Cuentas Fiscales». Documento de Trabajo 188. Santiago de Chile: Pontificia Universidad de Chile.

\section{BIBLIOGRAFÍA}

Aizenman, J.; Gavin, M. y Hausmann, R. (1996): «Optimal Tax Policy with Endogeneous Borrowing Constraint». Working Paper 5.558: NBER.

Aкitoby, B.; Clements, B.; Gupta, S. y Inchauste, G. (2006): «Public Spending, voracity, and Wagner's Law in Developing Countries». Journal of Political Economy 22, pp. 908-924.

Akitoby, B.; Clements, B.; Gupta, S. y Inchauste, G. (2004): «The Cyclical and Long-Term Behavior of Government Expenditures in Developing Countries». Working Paper 04/202: International Monetary Fund.

Alesina, A.; Campante, F. y Tabellini, G. (2007): «Why is fiscal policy often procyclical?». Working Paper Series: Harvard University.

Álvarez, J.; Bértola, L. y Porcile, G. (comp.) (2007): Primos ricos y empobrecidos: crecimiento, distribución del ingreso e instituciones en Australia-Nueva Zelanda vs. Argentina-Uruguay. Montevideo: Fin de Siglo.

Azar, P.; Bertino, M.; Bertoni, R.; Fleitas, S.; García, U.; Sanguinetti, C.; Sienra, M. y Torrelli, M. (2009): ¿De quiénes, para quiénes y para qué? Las finanzas públicas en el Uruguay del siglo XX. Montevideo: CSIC-UDELAR y Fin de Siglo.

Azar, P. y Fleitas, S. (2010): "Gestión fiscal del gasto social: una aproximación a la experiencia de diferentes tipos de "estado de bienestar" en el largo plazo». Ponencia presentada en las XXV Jornadas Anuales de Economía del Banco Central del Uruguay. Octubre de 2010.

Bértola, L. y LoRenzo, F. (2004): «Witches in the South: Kuznets-like swings in Argentina, Brazil and Uruguay since the 1870s», en L. Van Zanden y 
S. Heikenen (eds), The Experience of Economic Growth. Amsterdam: Aksant, pp. 299-320.

CAL, I. (2004): «El Papel de la Política en el Desempeño Económico de Uruguay de largo plazo: 1920-2002». Trabajo Monográfico, Facultad de Administración y Ciencias Sociales. Montevideo: Universidad ORT Uruguay.

Carbajal, F. y De Melo, G. (2007): «Volatilidad cíclica y arquitectura financiera doméstica, un estudio histórico comparado. El caso de Uruguay y Nueva Zelanda», en J. Álvarez; L. Bértola y G. Porcile (comps.), Primos Ricos y Empobrecidos. Crecimiento, distribución del ingreso e instituciones en Australia-Nueva Zelanda vs. Argentina Uruguay. Montevideo: Fin de Siglo, pp. 307-341.

CEPAL (2007): Panorama Social de América Latina y el Caribe. Santiago de Chile: CEPAL, Naciones Unidas.

CHANG, T. (2002): "An econometric test of Wagner's law for six countries based on co-integration and error-correction modeling techniques». Applied Economics 34, pp. 1157-1169.

Clemente, J.; Montañés, A. y Reyes, M. (1998): «Testing for Unit Roots in Variables with a Double Change in the Mean». Economics Letters 59, pp. 175-182.

Clements, B.; Faircloth, C. y Verhoeven, M. (2007): «Public Expenditure in Latin America: Trends and Key Policy Issues». Working Paper 07/21: International Monetary Fund.

Comín, F.; Díaz Fuentes, D. y Revuelta, J. (2008): «La fiscalidad comparada en América Latina y España, siglos XIX y XX». Ponencia presentada al IX Congreso de la Asociación Española de Historia Económica, Murcia.

Cominetti, R. (1994): «Ajuste Fiscal y Gasto Social». Revista de la CEPAL 54, pp. 47-60.

DíAz Fuentes, D. (1994): «Las políticas fiscales latinoamericanas frente a la gran depresión. Argentina, Brasil y México (1920-1940)». Madrid: Instituto de Estudios Fiscales.

Ffrench-Davis, R. (2003): "Financial crises and national policy issues: an overview». Serie Informes y Estudios Especiales. Oficina del Secretario General de la CEPAL.

FilgueIRA, F. (1998): «El nuevo modelo de prestaciones sociales en América Latina: residualismo y ciudadanía estratificada», en B. Roberts (ed.), Ciudadanía y política social. San José de Costa Rica: FLACSO/SSRC, pp. 71-116.

Gavin, M. y Perroti, R. (1997a): «Fiscal Policy in Latin America» NBER Macroeconomics Annual. Cambridge, Mass: MIT Press, pp. 11-61.

Gavin, M. y Perroti, R. (1997b): «Fiscal Policy and Saving in Good Times and Bad Times", en R. Hausmann y H. Reisen (eds), Promoting Savings in Latin America. París: IABD-OCDE.

Gavin, M.; Hausmann, R.; Perotti, R. y Talvi, E. (1996): «Managing Fiscal Policy in Latin America and the Caribbean: Volatility, Procyclicality, and 
Creditworthiness». Working Paper 326: Inter-American Development Bank, Office of the Chief Economist.

Hayo, B. (1994): «No further evidence on Wagner's law for Mexico». Public Finance 49, pp. 287-294.

Hicks, Q. y Wodon, N. (2001): «Protección social para los pobres en América Latina». Revista de la CEPAL 73, pp. 95-116.

Hodrick, R. y PREscotT, E. (1997): «Postwar U.S. Business Cycles: An Empirical Investigation». Journal of Money, Credit, and Banking 29 (1), pp. 1-16.

IlzetzKi, E. y Vegh, C. (2008): «Procyclical fiscal policy in developing countries: Truth or fiction?». Working Paper 1.419:NBER.

Johansen, S. (1991): «Estimation and Hypothesis Testing of Cointegration Vectors in Gaussian Vector Autorregresive Models». Econometrica 59, pp. 1.551-1.580.

Kamil, H. y Lorenzo, F. (1998): «Caracterización de las Fluctuaciones Cíclicas en la Economía Uruguaya». Montevideo: Facultad de Ciencias Económicas, Universidad de la República (mimeo).

Kaminsky, G., Reinhart, C. y Végh, C. (2004): "When it Rains, it Pours: Procyclical Capital Flows and Macroeconomic Policies». Working Paper 10.780: NBER.

Lane, P. (2003): «The Cyclical Behaviour of Fiscal Policy: Evidence from the OECD».

Lane, P. y ToRnell, A. (1999): «The voracity effect». American Economic Review 89, pp. 22-46.

Lindert, P. (2004): Growing Public: Social Spending and Economic Growth since the Eighteenth Century. Cambridge: Cambridge University Press.

Mailhos, S. y Sosa, S. (2000): «El Comportamiento Cíclico de la Política Fiscal en Uruguay». presentado en Jornadas Anuales de Economía del BCU.

Mailhos, S. y, Sosa, S. (1999): El Comportamiento Cíclico de la Política Fiscal en Uruguay, Monografía de Grado de la Licenciatura en Economía, Universidad de la República: Montevideo.

Martínez, R. y EspíndolA, E. (2007): «Gasto social en América Latina: una propuesta para su análisis». Borrador para la discusión. Preparado para la reunión técnica «La medición del gasto social: avances y desafíos metodológicos», Santiago 9-10 de agosto de 2007, División de Desarrollo Social, CEPAL.

Martner, R. y Tromben, V. (2003): «Tax reforms and fiscal stabilisation in Latin America». Documento presentado para Quinto taller de Banca d'Italia sobre Finanzas Públicas en Perugia, 3 al 5 de abril de 2003.

Martner, R. y Aldunate, E. (2006): «Política fiscal y protección social». Serie Gestión Pública 53: CEPAL-ILPES.

Mesa-Lago, C. (1986): «Introducción», en C. Mesa Lago (ed.), La crisis de la seguridad social y la atención a la salud. México: Fondo de Cultura Económica.

Murthy, N. R. V. (1993): «Further evidence on Wagner's law for Mexico: an application of cointegration analysis». Public Finance 48, pp. 77-85. 
Ocampo, J. A. (2002): "Developing Countries' Anti-Cyclical Policies in a Globalized World», en A. Dutt y J. Ros (eds), Development Economics and Structuralist Macroeconomics: Essays in Honour of Lance Taylor. London: Aldershot.

OdDone, G. (2005): «El largo declive económico de Uruguay durante el siglo XX». Tesis doctoral. Universitat de Barcelona, Departament d'Història i Institucions Econòmiques. Programa de Doctorado de Historia Económica, bienio 1997-1999.

Peacock, A. y Scott, A. (2000): «The curious attraction of Wagner's law». Public Choice 102, pp. 1-17.

Ravn, M. y Uhlig, H. (2002): «On adjusting the Hodrick-Prescott filter for the frequency of observations». The Review of Economics and Statistics 84 (2), pp. 371-375.

SEgura-Ubiergo, A. (2007): The political economy of the welfare state in Latin America: Globalization, democracy and development. New York: Cambridge University Press.

Stein, E.; Talvi, E. y Grisanti, A. (1998): Institutional Arrangements and Fiscal Performance: The Latin American Experience. Working Paper 367, InterAmerican Development Bank, Office of the Chief Economist.

Talvi, E. y Vegh, C. (2000): «Tax Base Variability and Procyclical Fiscal Policy». Working Paper 7.499: NBER.

Thornton, J. (1998): «The growth of public expenditure in Latin America: a test of Wagner's law». Cuadernos de Economía. Santiago de Chile: Pontificia Universidad Católica de Chile, pp. 255-263.

WAGner, A. H. (1912): Les fondements de l'economie politique. Vol. III. Paris: Girard y Brière.

WibBELs, E. (2006): «Dependency Revisited: International Markets, Business Cycles, and Social Spending in the Developing World», University of Washington.

Zivot, E. y ANDREws, D. W. K. (1992): «Further evidence of great crash, the oil price shock and unit root hypothesis». Journal of Business and Economic Statistics 10, pp. 251-270.

\section{APÉNDICE}

Para evaluar la interacción entre el gasto público y el producto se realizó un análisis de cointegración a partir del método de Johansen (1991). Según éste, verificada la condición de estacionariedad de las series (o sea que las mismas no presenten raíces unitarias) se estimaron los Vectores Autorregresivos, VAR (donde cada una de las variables se representa como función de los rezagos propios y de las demás variables). Esta especificación permite determinar el número de rezagos óptimo de las variables que hay que incluir, así como las intervenciones y variables determinísticas. Luego, se estima un modelo VECM (Vector de Mecanismo de Corrección 
de Error) que incorpora las relaciones de largo plazo entre las variables y el ajuste al equilibrio en el corto plazo, y se realiza el Test de Cointegración de Johansen.

El modelo general (VECM) tiene la forma:

$\Delta \mathrm{X}_{\mathrm{t}}=\mathrm{A}_{\mathrm{i}} \Delta \mathrm{X}_{\mathrm{it}-1}+\ldots+\mathrm{A}_{\mathrm{k}} \Delta \mathrm{X}_{\mathrm{it}-1-\mathrm{k}-1}+\Pi \mathrm{X}_{\mathrm{it}-\mathrm{k}}+\mu \mathrm{D}_{\mathrm{t}}+\Gamma+\varepsilon_{\mathrm{t}}$

donde $\varepsilon=\mathrm{N}\left(0, \sigma^{2}\right)$,

$\mu$ es un vector de constantes,

$\mathrm{X}$ es el vector de variables endógenas (en este caso: $\left[\begin{array}{l}G t] \\ Y_{t}\end{array}\right]$ ),

y $\mathrm{D}$ es el conjunto de intervenciones (variables dummies)

La información sobre las relaciones de largo plazo está contenida en la matriz $\Pi=\alpha \beta^{\prime}$.

El rango de la matriz $\Pi$ indica el número de relaciones de cointegración: $\beta$ es el vector de coeficientes de las relaciones de largo plazo y $\alpha$ es el vector de los mecanismos de ajuste al equilibrio de largo plazo.

El procedimiento se inicia chequeando la presencia de raíces unitarias en las series. Para el conjunto de países considerado, la aplicación del test de Dickey-Fuller Aumentado (ADF) no permitió rechazar la existencia de raíces unitarias en los niveles de las variables, pero la descartó en las diferencias, por lo que las series son integradas de orden 1: I(1).

Debido a las limitaciones de los test ADF en presencia de cambios estructurales se realizaron 2 test adicionales: Zivot y Andrews (1992), que permite un cambio estructural en la serie, y Clemente, Montañés y Reyes (1998), que admite dos cambios estructurales. Estas metodologías alternativas a los ADF rechazan la presencia de raíz unitaria en las diferencias, pero no permiten rechazar la existencia de una raíz unitaria en los niveles. En todos los casos se consideró una cantidad de rezagos suficientes para que los residuos de las ecuaciones de la prueba de raíz unitaria se distribuyeran normales.

En el modelo estimado, la cantidad de rezagos se determinó automáticamente por el criterio de Akaike y se evaluó la ausencia de autocorrelación serial en los residuos y su distribución normal conjunta y de cada ecuación.

Para probar la existencia de relaciones de largo plazo entre las variables, se estimó el modelo VECM, considerando la información sobre rezagos obtenida en el paso anterior. Los resultados del test de cointegración de Johansen (prueba de la traza y de valores propios de la matriz П) no permiten rechazar la existencia de un vector de cointegración al 5\% de significación.

Para la relación de largo plazo estimada, se testeó la exclusión de las variables (que evalúa que el coeficiente $\beta$ sea significativamente diferente de cero) y el contraste de exogeneidad débil, a través de la prueba de hipótesis $\alpha=0$. El resultado fue el rechazo de la hipótesis nula $\alpha=0$ para el caso del 
CUADRO A-1

ESTADÍSTICAS DESCRIPTIVAS DE LAS SERIES UTILIZADAS PARA CADA PAÍS, 1900 y 2000

\begin{tabular}{|c|c|c|c|c|c|c|c|c|c|}
\hline \multirow[b]{2}{*}{ Países } & \multicolumn{4}{|c|}{ Tasas de crecimiento } & \multicolumn{5}{|c|}{ Gasto Gobierno Central (GC) sobre PBI } \\
\hline & PBI & $\begin{array}{c}\text { Gasto } \\
\text { GC }\end{array}$ & $\begin{array}{l}\text { PBI per } \\
\text { cápita }\end{array}$ & $\begin{array}{c}\text { Gasto GC } \\
\text { per cápita }\end{array}$ & En 1900 & En 2000 & Media & $\begin{array}{l}\text { Desvío } \\
\text { estándar }\end{array}$ & $\begin{array}{l}\text { Coeficiente } \\
\text { de variación }\end{array}$ \\
\hline España & $3,00 \%$ & $4,11 \%$ & $2,23 \%$ & $3,32 \%$ & $9,06 \%$ & $26,24 \%$ & $14,50 \%$ & $7,21 \%$ & $49,68 \%$ \\
\hline Brasil & $4,48 \%$ & $5,52 \%$ & $2,15 \%$ & $3,17 \%$ & $7,31 \%$ & $19,69 \%$ & $11,00 \%$ & $3,75 \%$ & $34,13 \%$ \\
\hline Argentina & $3,20 \%$ & $3,69 \%$ & $1,08 \%$ & $1,55 \%$ & $10,59 \%$ & $16,97 \%$ & $11,52 \%$ & $3,31 \%$ & $28,72 \%$ \\
\hline Chile & $3,46 \%$ & $4,16 \%$ & $1,79 \%$ & $2,47 \%$ & $10,54 \%$ & $20,62 \%$ & $16,63 \%$ & $6,94 \%$ & $41,72 \%$ \\
\hline Canadá & $3,86 \%$ & $5,01 \%$ & $2,07 \%$ & $3,20 \%$ & $6,35 \%$ & $18,98 \%$ & $17,44 \%$ & $8,86 \%$ & $50,81 \%$ \\
\hline Nueva Zelanda & $2,99 \%$ & $3,48 \%$ & $1,41 \%$ & $1,89 \%$ & $13,41 \%$ & $21,44 \%$ & $17,39 \%$ & $5,11 \%$ & $29,36 \%$ \\
\hline Italia & $2,95 \%$ & $3,76 \%$ & $2,40 \%$ & $3,21 \%$ & $13,10 \%$ & $28,80 \%$ & $27,21 \%$ & $10,83 \%$ & $39,79 \%$ \\
\hline Uruguay & $2,64 \%$ & $3,08 \%$ & $1,38 \%$ & $1,81 \%$ & $10,78 \%$ & $23,00 \%$ & $14,00 \%$ & $3,71 \%$ & $26,71 \%$ \\
\hline $\begin{array}{l}\text { Uruguay-GC } \\
\text { Consolidado }\end{array}$ & $2,64 \%$ & $1,35 \%$ & $1,38 \%$ & $0,10 \%$ & $11,83 \%$ & $38,11 \%$ & $20,09 \%$ & $7,55 \%$ & $37,60 \%$ \\
\hline
\end{tabular}

Fuentes: Elaborado a partir de datos de los apartados 2 y 3 . 
CUADRO A-2

DESVÍO ESTÁNDAR Y CORRELACIÓN ENTRE COMPONENTES CÍCLICOS DE LAS VARIABLES FISCALES Y DEL PRODUCTO, 1900 a 2000

\begin{tabular}{|l|c|c|c|}
\hline \multirow{2}{*}{ Países } & \multicolumn{3}{|c|}{ Gasto Público Gobierno Central (componente cíclico) } \\
\cline { 2 - 4 } España & Desvío estándar & Correlación con el PBI (c. c.) & P-Valor \\
\hline Brasil & 0,0902 & 0,0565 & 0,5946 \\
\hline Argentina & 0,1092 & 0,0789 & 0,4572 \\
\hline Chile & 0,1107 & $0,2176^{* * *}$ & 0,0383 \\
\hline Canadá & 0,0814 & $0,4480^{* * *}$ & 0,0001 \\
\hline Nueva Zelanda & 0,1186 & $0,2845^{* * *}$ & 0,0063 \\
\hline Italia & 0,0837 & $0,2277^{* *}$ & 0,0299 \\
\hline
\end{tabular}

Notas: nivel de significación expresado para $10 \%(*), 5 \%(* *)$ y $1 \%(* * *)$. Para las estimaciones se utilizó el filtro de Hodrik y Prescott (1997) con un parámetro de suavizado de 6,25 tal como recomiendan Ravn y Uhlig (2002) para datos anuales. De cualquier manera, las conclusiones se mantienen cuando se utiliza el parámetro de suavizado igual a 100 tal como recomendaban Hodrik y Prescott.

gasto, pero no para el producto. Por lo tanto, el gasto es la variable que se ajusta período a período a los desvíos de la relación de equilibrio de largo plazo, siendo el producto débilmente exógeno en la relación de cointegración, lo cual permite entender la relación de cointegración como una ecuación de elasticidad. 
CUADRO A-3

FASES DE AJUSTE Y DESAJUSTE FISCAL E INTENSIDAD Y MAGNITUD QUE ALCANZAN

(En porcentaje del producto)

\begin{tabular}{|c|c|c|c|c|c|c|}
\hline \multicolumn{3}{|c|}{ FASES DE AJUSTE } & \multicolumn{3}{|c|}{ Variación del Gasto Social } & \multirow[b]{2}{*}{ Variación del PBI } \\
\hline Período & Intensidad del ajuste & Magnitud del ajuste & ... en educación & $\ldots$ en salud & ...en seg. social & \\
\hline 1910-1911 & 0,19 & 0,7 & $14 \%$ & $22 \%$ & $6 \%$ & $-3 \%$ \\
\hline 1915-1918 & $-0,44$ & 4,5 & $-4 \%(*)$ & $0 \%(*)$ & $7 \%(*)$ & $9 \%$ \\
\hline $1922-1924$ & 0,3 & 2,3 & $18 \%(*)$ & $52 \%$ & $-22 \%(*)$ & $24 \%$ \\
\hline 1926-1927 & 1,6 & 1,7 & $18 \%(*)$ & $23 \%$ & $5 \%(*)$ & $19 \%$ \\
\hline 1946-1947 & $-0,78$ & 3,2 & $-7 \%(*)$ & $-14 \%(*)$ & $20 \%$ & $12 \%$ \\
\hline 1950-1951 & $-0,88$ & 1,3 & $17 \%(*)$ & $38 \%$ & $32 \%$ & $23 \%$ \\
\hline $1955-1959$ & 1,58 & 3,6 & $-24 \%(*)$ & $-34 \%(*)$ & $-7 \%(*)$ & $0 \%$ \\
\hline $1963-1964$ & $-0,99$ & 2,8 & $-22 \%(*)$ & $-19 \%(*)$ & $-20 \%(*)$ & $3 \%$ \\
\hline $1972-1973$ & $-1,42$ & 4,4 & $-17 \%(*)$ & $-18 \%(*)$ & $-20 \%(*)$ & $0 \%$ \\
\hline 1976-1977 & $-1,25$ & 3,7 & $18 \%$ & $39 \%$ & $20 \%$ & $5 \%$ \\
\hline 1985-1986 & $-1,27$ & 4,5 & $19 \%$ & $51 \%$ & $-24 \%(*)$ & $10 \%$ \\
\hline
\end{tabular}


CUADRO A-3 (Cont.)

\begin{tabular}{|c|c|c|c|c|c|c|}
\hline \multicolumn{3}{|c|}{ FASES DE AJUSTE } & \multicolumn{3}{|c|}{ Variación del Gasto Social } & \multirow[b]{2}{*}{ Variación del PB } \\
\hline Período & Intensidad del ajuste & Magnitud del ajuste & ... en educación & ... en salud & ...en seg. social & \\
\hline 1990-1991 & 0,36 & 3,5 & $-5 \%(*)$ & $6 \%$ & $19 \%$ & $4 \%$ \\
\hline 1996-1998 & $-1,17$ & 0,8 & $102 \%$ & $20 \%$ & $24 \%$ & $16 \%$ \\
\hline \multicolumn{3}{|c|}{ FASES DE DESAJUSTE } & \multicolumn{3}{|c|}{ Variación del Gasto Social } & \\
\hline Período & $\begin{array}{c}\text { Intensidad del } \\
\text { desajuste }\end{array}$ & $\begin{array}{c}\text { Magnitud del } \\
\text { desajuste }\end{array}$ & $\begin{array}{c}\ldots \text { en educa- } \\
\text { ción }\end{array}$ & ... en salud & ...en seg. social & $\begin{array}{c}\text { Variación del } \\
\text { PBI } \\
\end{array}$ \\
\hline 1912-1914 & $-4,93$ & $-5,1$ & $-5 \%$ & $52 \%$ & $2 \%$ & $-9 \%$ \\
\hline 1919-1921 & $-1,98$ & $-1,5$ & $25 \%$ & $59 \%$ & $49 \%$ & $4 \%$ \\
\hline 1930-1933 & $-1,29$ & $-1,4$ & $5 \%$ & $-34 \%(*)$ & $90 \%$ & $-12 \%$ \\
\hline 1937-1939 & $-2,42$ & $-2,5$ & $-1 \%(*)$ & $-7 \%(*)$ & $6 \%(*)$ & $13 \%$ \\
\hline 1952-1954 & $-2,05$ & $-1,2$ & $8 \%(*)$ & $15 \%$ & $39 \%$ & $13 \%$ \\
\hline 1960-1962 & $-3,75$ & $-5,3$ & $142 \%$ & $66 \%$ & $84 \%$ & $4 \%$ \\
\hline 1974-1975 & $-4,97$ & $-3,6$ & $-10 \%$ & $-19 \%(*)$ & $71 \%$ & $9 \%$ \\
\hline 1980-1982 & $-8,71$ & -9 & $32 \%$ & $22 \%$ & $63 \%$ & $-2 \%$ \\
\hline 1987-1989 & $-3,12$ & $-0,3$ & $8 \%$ & $17 \%$ & $20 \%$ & $11 \%$ \\
\hline 1992-1995 & $-1,94$ & $-2,3$ & $5 \%$ & $43 \%$ & $39 \%$ & $17 \%$ \\
\hline 1999-2000 & $-4,03$ & $-2,9$ & $-15 \%$ & $1 \%$ & $3 \%$ & $-4 \%$ \\
\hline
\end{tabular}

(*) Comportamiento «pro ajuste» que representa una reducción del gasto social o un aumento del gasto social proporcionalmente menor al del PBI. 
Reproduced with permission of the copyright owner. Further reproduction prohibited without permission. 